\title{
Microencapsulation of Lactobacillus rhamnosus GG with Resistant Starch and Xanthan Gum
}

\author{
Pei Ying HOH, Ka Wai LAI, Yu Hsuan HOW and Liew Phing PUI \\ Department of Food Science and Nutrition, Faculty of Applied Sciences, UCSI University, \\ Cheras, Wilayah Persekutuan Kuala Lumpur, Malaysia
}

('Corresponding author's e-mail: puilp@ucsiuniversity.edu.my)

Received: 6 March 2020, Revised: 19 March 2021, Accepted: 26 March 2021

\begin{abstract}
Microencapsulation is the most common method in improving probiotic survivability against adverse conditions. In this research, resistant starch was incorporated as prebiotic coating material during the microencapsulation of Lactobacillus rhamnosus GG (LGG) in calcium alginate beads coated with xanthan gum. Three types of microcapsules were produced: LGG only, LGG with resistant starch, and LGG with resistant starch and xanthan gum coating. The size and morphology of microcapsules were measured. Furthermore, the viability for free cells and microencapsulated LGG was tested in the simulated gastric juice (SGJ) ( $\mathrm{pH} \mathrm{2.0,2} \mathrm{h)} \mathrm{and} \mathrm{simulated} \mathrm{intestinal} \mathrm{juice} \mathrm{(SIJ)} \mathrm{(pH} \mathrm{7.5,} 4 \mathrm{~h})$. The results indicated that $2.0 \%(\mathrm{w} / \mathrm{v})$ resistant starch and $0.3 \%(\mathrm{w} / \mathrm{v})$ xanthan gum had the highest microencapsulation efficiency (MEE). The morphology for microencapsulated LGG was spherical and white. The mean diameter for all 3 types of microcapsules was in between the range of 562.67 to 614.33 $\mu \mathrm{m}$ and xanthan gum-coated microcapsules had the highest MEE of $84.67 \%$. The addition of resistant starch and xanthan gum had increased the MEE for encapsulated probiotics. Both encapsulated LGG with and without xanthan gum coating had higher survivability than free cells, which indicated the positive role of resistant starch and xanthan gum in promoting the viability of probiotics during gastrointestinal transit. In general, co-extrusion encapsulation and the addition of resistant starch and xanthan gum coating could protect the viable LGG against the harsh human gastrointestinal condition.
\end{abstract}

Keywords: L. rhamnosus GG, Microencapsulation, Probiotics, Resistant starch, Xanthan gum

\section{Introduction}

The development of functional foods has become a new trend in the food processing industry. The incorporation of probiotics into food products is considered functional food and it is also classified as a microbial food supplement. According to Krasaekoopt and Watcharapoka, probiotics can confer health benefits when being consumed at a recommended amount of $10^{6}$ to $10^{7} \mathrm{CFU} / \mathrm{mL}$ [1]. Some of the health benefits include suppressing cancer disease, improving skin health, reducing serum cholesterol, improving gut microbiota balance, and enhancing body defense mechanism against pathogens [2,3].

LGG is a gram-positive lactic acid bacteria (LAB) strain which belong to the Lactobacillus rhamnosus species [4]. It was $1^{\text {st }}$ being isolated from the human fecal samples and it is currently being widely studied across the world due to its potential health benefits. The consumption of LGG probiotic supplements has been found to have an antagonistic effect against pathogens such as S. sonnei, Staphylococcus, and Streptococcus strains [5]. It also helped in the treatment of gastrointestinal infections and reduction in constipation status [6].

Prebiotics, defined as short-chain carbohydrates (SCCs), are non-digestible food ingredients that can improve host health by stimulating the growth or activity of bacteria in the colon [7]. Galactooligosaccharides (GOS), fructooligosaccharides (FOS), inulin, and resistant starch are some of the 
http://wjst.wu.ac.th

examples of prebiotics [8]. According to literature, the combination of prebiotic and probiotic, also known as "synbiotic", has shown synergistic health benefits to humans such as improving probiotic proliferation in the gut, lowering inflammation and total cholesterol $[9,10]$.

However, for probiotics to exert health benefits, they must maintain high viability during gastrointestinal transit [11]. In addition, other factors such as extreme $\mathrm{pH}$, high food processing temperature, water activity and prolong storage of food products will also reduce the viability of probiotics [12]. Therefore, technologies such as microencapsulation can help to provide effective protection to probiotics by separating them from the external environment, and thus, it can maintain the viability of probiotics $[11,13]$. Co-extrusion is one of the microencapsulation technologies which utilizes vibration nozzle technology to produce a laminar liquid jet [14]. Encapsulation of probiotics using co-the extrusion method had shown to extend the viability of probiotics [15].

Various wall materials like alginate, carboxymethyl cellulose (CMC), chitosan, carrageenan, and gelatin have been incorporated during the microencapsulation process as a way to protect the core material [16]. Alginate, a linear anionic polysaccharide that is derived from different types of algae and is the most commonly used wall material for microencapsulation. Alginate is made up of two structural units which are the D-mannuronic acid and L-guluronic acid, both units are joined together by glycosidic bonds. It is commonly chosen as shell wall material due to its biocompatibility, low cost, and non-toxic properties $[17,18]$.

Furthermore, calcium alginate beads are commonly coated with polymers such as chitosan, poly Llysine (PLL), xanthan gum, and whey protein [19]. The coating of alginate beads can improve the stability of microcapsules and thus, increasing the viability of probiotics [20]. Xanthan gum, an anionic microbial exopolysaccharide, is used as food thickener as it is easily dispersed in cold and hot water and possess high viscosities at low concentration [21,22]. Studies had found that xanthan gum can protect probiotics in acidic environments [23].

To date, emulsion, extrusion, and spray drying are the most common methods used for the encapsulation of probiotics [1]. However, the research on the production of microcapsules using coextrusion is scarce. Furthermore, the combined effect of resistant starch as prebiotic and xanthan gum as a coating material for the LGG microcapsules has yet to be explored. Hence, the effect of resistant starch and xanthan gum coating on the viability of LGG microencapsulated with sodium alginate using coextrusion microencapsulation against simulated gastro-intestinal conditions was evaluated in this study.

\section{Materials and methods}

\section{Materials}

LGG (ATCC 53103) capsules were purchased from a local pharmacy. Hi-maize 260 resistant starch was purchased from Salisbury, USA. Xanthan gum powder (Sigma Aldrich, USA) was used as a coating material. Sodium alginate and calcium chloride were purchased from R\&M Chemicals, UK to produce microbeads. Pepsin (HmbG chemicals, Germany) and bile salts (R\&M Chemicals, UK) were used to prepare simulated gastric and intestinal juices. MRS agar and MRS broth were purchased from Oxoid, UK and used for microbiological analysis.

\section{Preparation of culture}

LGG was inoculated into De Man Rogosa Sharpe (MRS) broth and incubated at $37{ }^{\circ} \mathrm{C}$ for $24 \mathrm{~h}$ under anaerobic conditions. The cells were harvested through centrifugation at $4200 \mathrm{rpm}, 4{ }^{\circ} \mathrm{C}$ for 10 min. After centrifugation, the supernatant was discarded while the cell pellets were re-suspended in 25 $\mathrm{mL}$ of phosphate-buffered solution (PBS) with a final concentration of $10^{8} \mathrm{CFU} / \mathrm{mL}$. The PBS was prepared by dissolving $0.795 \mathrm{~g}$ of disodium hydrogen phosphate, $0.144 \mathrm{~g}$ of potassium dihydrogen phosphate, and $9 \mathrm{~g}$ of sodium chloride into $1 \mathrm{~L}$ of sterile distilled water and sterilized at $121{ }^{\circ} \mathrm{C}$ for 15 $\min$. 
http://wjst.wu.ac.th

\section{Preparation of resistant starch solution}

The resistant starch solution was prepared according to Zanjani et al. [17] with modification. Two g of resistant starch powder was added to $100 \mathrm{~mL}$ distilled water and was heated until all starch granules had been gelatinized. The alginate-starch solution was prepared by slowly adding sterile $2.0 \%(\mathrm{w} / \mathrm{v})$ resistant starch solution into sterile $1.5 \%(\mathrm{w} / \mathrm{v})$ sodium alginate solution at a volume ratio of $1: 1$, the solution was mixed gently to obtain a homogenous shell solution.

\section{Microencapsulation process}

Microencapsulation of LGG was carried out using Büchi Encapsulator B-390 (Büchi Labortechnik AG, Flawil, Switzerland) through the co-extrusion method as described by Chew et al. [24] with modification. All glass wares were sterilized by autoclaving at $121{ }^{\circ} \mathrm{C}$ for $15 \mathrm{~min}$ before conducting the microencapsulation process. The core fluid (LGG suspension in PBS solution) and the shell fluid (sodium alginate solution or alginate-starch solution) were transferred into 2 separate pressured bottles that were connected to the Büchi Encapsulator B-390. During the microencapsulation process, core fluid and shell fluid were pumped simultaneously through the concentric nozzles with a diameter of $150 \mu \mathrm{m}$ (inner nozzle) and $300 \mu \mathrm{m}$ (shell nozzle) by the air pressure of 500 mbar to produce a core-shell fluid stream. The vibration frequency of the nozzle was set at $300 \mathrm{~Hz}$ with an amplitude of 3 and a voltage of $1.5 \mathrm{kV}$. The microcapsules were collected in calcium chloride solution under magnetic stirring for $30 \mathrm{~min}$. The microcapsules were rinsed with PBS and kept in a chiller at $4{ }^{\circ} \mathrm{C}$ before further usage.

\section{Coating microcapsules with xanthan gum}

The preparation of the xanthan gum solution was adapted from Shu et al. [25] with modification. Fifteen $g$ of microcapsules were transferred to $100 \mathrm{~mL}$ of $0.3 \%(\mathrm{w} / \mathrm{v})$ xanthan gum solution and stirred at $100 \mathrm{rpm}$ for $30 \mathrm{~min}$ at room temperature to produce xanthan gum-coated microcapsules. The coated microcapsules were dried with filter paper and kept in a chiller at $4{ }^{\circ} \mathrm{C}$ before further usage.

\section{Optimizing concentration of resistant starch and xanthan gum}

Optimization of wall material and coating material for microencapsulation of LGG was performed. For resistant starch optimization, Hi-maize 260 resistant starch was incorporated into $1.5 \%$ (w/v) sodium alginate after gelatinization. The microencapsulation process was repeated from 0 to $2 \%(\mathrm{w} / \mathrm{v})$ resistant starch.

To optimize the concentration of xanthan gum coating, microencapsulation was conducted using the chosen concentration of resistant starch. After hardening in calcium chloride solution, the beads were coated in different concentrations of xanthan gum $(0.1$ and $0.3 \% \mathrm{w} / \mathrm{v})$. The optimum concentration for resistant starch and xanthan gum were chosen based on the size of the bead and MEE [2].

Microencapsulation efficiency $(\%)=\left(\mathrm{N} / \mathrm{N}_{0}\right) \times 100$

where: $\mathrm{N}=$ number of viable entrapped cells $(\mathrm{CFU} / \mathrm{g})$ released from the microcapsules

$\mathrm{N}_{0}=$ number of free viable cells $(\mathrm{CFU} / \mathrm{mL})$ in the culture

\section{Morphology and size of beads}

The diameters and morphology of 25 randomly selected beads were observed using an optical microscope (CX23, Olympus, Japan), with $\times 100$ magnification. The beads were placed onto the calibrated micrometer scale to measure the size of beads [26].

\section{Release of entrapped bacteria}

Prior to cell enumeration, the solubilization of co-encapsulated LGG cells was adapted from Chávarri et al. [27] with modification. For the decomposition of the capsules, $1 \mathrm{~g}$ of beads was added into $9 \mathrm{~mL}$ of $1.0 \%(\mathrm{w} / \mathrm{v})$ sterile tri-sodium citrate solution and it was mixed through vortexing until all beads were decomposed. For cell enumeration, $1 \mathrm{~mL}$ of the decomposed beads mixture or $1 \mathrm{~mL}$ of free bacteria was serially diluted with PBS. The drop plate method was used for the enumeration of bacteria whereby 
http://wjst.wu.ac.th

$0.01 \mathrm{~mL}$ of diluents were dropped onto MRS agar using a micropipette. The droplets were allowed to dry before the plates were sealed with parafilm and incubated at $37{ }^{\circ} \mathrm{C}$ for $48 \mathrm{~h}$ in an upside-down position. All samples were enumerated in triplicates. The viable cell counts were expressed in colony-forming unit per milliliter $(\mathrm{CFU} / \mathrm{mL})$ and were then converted into $\log \mathrm{CFU} / \mathrm{mL}$.

Survival of free cell and encapsulated bacteria with or without xanthan gum coating afterincubation in the simulated digestive system

SGJ and SIJ were prepared according to Chia et al. [16] with modification. SGJ was prepared by mixing $3.5 \mathrm{~mL}$ of $0.1 \mathrm{M}$ hydrochloric acid $(\mathrm{HCl})$ and $1 \mathrm{~g}$ of sodium chloride in $500 \mathrm{~mL}$ of distilled water. The solution was adjusted to $\mathrm{pH} 2.0 \mathrm{using} 1.0 \mathrm{M} \mathrm{HCl}$, the solution was sterilized at $121^{\circ} \mathrm{C}$ for $15 \mathrm{~min}$ and cooled to room temperature before adding $1.6 \mathrm{~g}$ of pepsin. SIJ was prepared by dissolving $3.4 \mathrm{~g}$ of potassium dihydrogen phosphate in $125 \mathrm{~mL}$ of distilled water, then, it was mixed with $95 \mathrm{~mL}$ of $0.1 \mathrm{M}$ $\mathrm{NaOH}$, following by topping up the solution to $500 \mathrm{~mL}$ with distilled water. The $\mathrm{pH}$ of the solution was adjusted to 7.5 using $1.0 \mathrm{M} \mathrm{NaOH}$ and the solution was sterilized at $121{ }^{\circ} \mathrm{C}$ for $15 \mathrm{~min}$ and cooled down to room temperature before adding $3 \mathrm{~g}$ of bile salt.

Sequential digestion was conducted according to Chia et al. [16] with slight modification. To evaluate the viability and survivability of LGG under simulated gastrointestinal conditions, $1 \mathrm{~g}$ of beads (with or without xanthan gum coating) or $1 \mathrm{~mL}$ of free cells were added to $9 \mathrm{~mL}$ of sterile $\mathrm{pH} 2.0 \mathrm{SGJ}$. It was then mixed at $100 \mathrm{rpm}$ while incubated at $37^{\circ} \mathrm{C}$ for $2 \mathrm{~h}$. After the $2^{\text {nd }}$ hour of incubation, the remaining tubes of beads or free cells were centrifuged and then transferred to $9 \mathrm{~mL}$ of SIJ and further incubated at $37^{\circ} \mathrm{C}$ for $4 \mathrm{~h}$. At $0,2,4$, and $6 \mathrm{~h}$ of exposure, the beads or free cells were removed from the incubator, centrifuged, and washed twice with PBS solution.

To evaluate the viability of LGG, the centrifuged microcapsules were added to $9 \mathrm{~mL}$ of $1 \%(\mathrm{w} / \mathrm{v})$ tri-sodium citrate solution followed by vortexing. For free cells, the cell pellets obtained from centrifugation were resuspended in $9 \mathrm{~mL}$ PBS. The mixture of microcapsules or free cells was serially diluted with PBS solution. An aliquot of $0.01 \mathrm{~mL}$ of the mixture was dropped onto MRS agar. The plates were sealed with parafilm and incubated at $37{ }^{\circ} \mathrm{C}$ for $48 \mathrm{~h}$ in an upside-down position. The viable cell counts for free cells and microcapsules were expressed as logarithm colony-forming unit per millimeter $(\log \mathrm{CFU} / \mathrm{mL})$ and logarithm colony forming unit per gram $(\log \mathrm{CFU} / \mathrm{g})$, respectively.

\section{Statistical analysis}

All analysis was conducted in triplicate and the results were expressed as mean \pm standard deviation. Data were analyzed using MINITAB 16 (Minitab, Inc, Pennsylvania, USA). One-way analysis of variance (ANOVA) was carried out, while the significant difference among the average values was analyzed using Tukey's post hoc test. The significant difference was set at $(p<0.05)$.

\section{Results and discussion}

Optimization of concentration of resistant starch and xanthan gum

For this optimization test, the concentration of sodium alginate was fixed at $1.5 \%(\mathrm{w} / \mathrm{v})$. As shown in Table 1, an increment in resistant starch concentration did not significantly increase the size of beads ( $p>0.05)$. This is in agreement with Etchepare et al. [28] who reported that the addition of gelatinized starch to alginate did not increase the bead size. For MEE, the addition of resistant starch improved the MEE for microencapsulated LGG. It was observed that alginate beads that had no resistant starch $(0$ $\%(\mathrm{w} / \mathrm{v}))$ had the lowest MEE (21.31\%). When gelatinized resistant starch was incorporated, it was observed that the MEE had been improved for both concentrations.

According to Sarao and Arora [29], single-layered alginate beads were found to be susceptible to disintegration under harsh chemical environments and the presence of chelating agents such as chloride ions. Cell leakage occurred due to high porosity in the alginate matrix when there was no addition of filler materials such as gelatinized starch. In addition, the survivability of encapsulated probiotics was increased when additional coating such as PLL or chitosan was added to alginate when tested under 
http://wjst.wu.ac.th

simulated gastrointestinal conditions [30]. This could explain the results of low MEE for microencapsulation LGG without the addition of resistant starch.

Table 1 Effect of different concentrations of resistant starch on bead size and MEE of microencapsulated LGG.

\begin{tabular}{cccc}
\hline $\begin{array}{c}\text { Alginate } \\
(\%, \mathbf{w} / \mathbf{v})\end{array}$ & $\begin{array}{c}\text { Resistant starch } \\
(\%, \mathbf{w} / \mathbf{v})\end{array}$ & $\begin{array}{c}\text { Diameter } \\
(\boldsymbol{\mu m})\end{array}$ & $\begin{array}{c}\text { Microencapsulation } \\
\text { efficiency (MEE) }(\%)\end{array}$ \\
\hline 1.5 & 0 & $625.33 \pm 60.27^{\mathrm{a}}$ & $21.31 \pm 1.28^{\mathrm{a}}$ \\
1.5 & 1 & $555.33 \pm 51.18^{\mathrm{a}}$ & $55.55 \pm 2.98^{\mathrm{b}}$ \\
1.5 & 2 & $617.47 \pm 49.65^{\mathrm{a}}$ & $74.72 \pm 2.68^{\mathrm{c}}$ \\
\hline
\end{tabular}

${ }^{\mathrm{a}-\mathrm{c}}$ means \pm standard deviations $(n=3)$ followed by different superscript letters within the same column are significantly different at $p \leq 0.05$ according to Tukey's test.

The results are in agreement with Zanjani et al. [11] who stated that the resistant starch had filled up the cavities of alginate capsules, thus, it helped to strengthen the alginate beads structure. A stronger alginate beads structure can reduce the amount of leakage of probiotics, thereby increasing the MEE. Resistant starch of $2 \%(\mathrm{w} / \mathrm{v})$ had the highest MEE of $74.72 \%$ among the 3 formulations. Hence, 2 $\%(\mathrm{w} / \mathrm{v})$ resistant starch was chosen as the optimum concentration to be incorporated for the microencapsulation of LGG.

The concentration of resistant starch was fixed at $2 \%(\mathrm{w} / \mathrm{v})$ in the previous optimization test, beads were produced using the fixed formulation $(1.5 \%(\mathrm{w} / \mathrm{v})$ alginate and $2.0 \%(\mathrm{w} / \mathrm{v})$ resistant starch) and further coated with different concentrations of xanthan gum. MEE was being compared among coated and uncoated microcapsules. From Table 2, the mean diameter for alginate-starch beads was $681.10 \mu \mathrm{m}$ which was smaller than 0.1 and $0.3 \%(\mathrm{w} / \mathrm{v})$ xanthan gum-coated microcapsules. When comparing the size of beads of 0.1 and $0.3 \%(\mathrm{w} / \mathrm{v})$ xanthan gum-coated microcapsules, it was shown that there was no significant difference $(p>0.05)$ among these 2 types of beads.

The result is in contrast with Zanjani et al. [17] findings whereby the addition of chitosan and PLL coatings to the microcapsules increased the size of beads. This could be due to the difference in the type and concentration of wall coating. The chemical interaction between xanthan gum coating and alginatestarch beads was not known as the research carried out on xanthan gum coating was scarce.

In addition, for MEE, there was no significant difference $(p>0.05)$ among $0.1 \%(\mathrm{w} / \mathrm{v})$ xanthan gum-coated and uncoated microcapsules. When the xanthan gum coating concentration had increased to $0.3 \%(\mathrm{w} / \mathrm{v})$, the MEE had been increased. Beads coated with $0.3 \%(\mathrm{w} / \mathrm{v})$ xanthan gum also had a high MEE of $87.92 \%$ which showed there was only little loss of LGG and the positive role of incorporation of resistant starch and xanthan gum coatings.

The result is not in agreement with Zanjani et al. [17] who had reported that the addition of coating had no significant difference $(p>0.05)$ on the MEE. The different findings in terms of MEE could be due to different microencapsulation methods and wall materials used, also, the type of probiotic strain will affect the effectiveness of microencapsulation [21]. The high MEE demonstrated by LGG microcapsules coated with $0.3 \%(\mathrm{w} / \mathrm{v})$ xanthan gum could be due to the reduction in polymer network space through the increase of xanthan gum coating concentration [31]. This could have strengthened the polymer network, hence providing better protection towards the probiotic LGG cells encapsulated within the xanthan-coated alginate polymer. The MEE for $0.3 \%(\mathrm{w} / \mathrm{v})$ xanthan gum coated beads was higher than $0.1 \%(\mathrm{w} / \mathrm{v})$ 
http://wjst.wu.ac.th

xanthan gum-coated and uncoated microcapsules (control). Therefore, $0.3 \%(\mathrm{w} / \mathrm{v})$ xanthan gum was chosen as the coating material for microencapsulated LGG.

Table 2 Effect of different concentrations of xanthan gum on bead size and MEE of xanthan gum-coated microencapsulated LGG.

\begin{tabular}{ccccc}
\hline $\begin{array}{c}\text { Alginate } \\
(\%, \mathbf{w} / \mathbf{v})\end{array}$ & $\begin{array}{c}\text { Resistant starch } \\
(\%, \mathbf{w} / \mathbf{v})\end{array}$ & $\begin{array}{c}\text { Xanthan gum } \\
(\%, \mathbf{w} / \mathbf{v})\end{array}$ & $\begin{array}{c}\text { Diameter } \\
(\boldsymbol{\mu m})\end{array}$ & $\begin{array}{c}\text { Microencapsulation } \\
\text { efficiency (MEE) } \\
(\%)\end{array}$ \\
\hline 1.5 & 2.0 & 0.0 & $681.10 \pm 20.61^{\mathrm{b}}$ & $73.32 \pm 1.08^{\mathrm{a}}$ \\
1.5 & 2.0 & 0.1 & $509.20 \pm 45.06^{\mathrm{a}}$ & $76.85 \pm 1.30^{\mathrm{a}}$ \\
1.5 & 2.0 & 0.3 & $545.73 \pm 15.65^{\mathrm{a}}$ & $87.92 \pm 2.47^{\mathrm{b}}$ \\
\hline
\end{tabular}

a-b means \pm standard deviations $(n=3)$ followed by different superscript letters within the same column are significantly different at $p \leq 0.05$ according to Tukey's test.

Effect of resistant starch and xanthan gum coating on the microencapsulation efficiencies and size and morphology of beads

From Figure 1, all microcapsules produced were white. The shape for 3 types of microcapsules was observed to be uniform and spherical shaped. All microcapsules also had smooth surfaces when viewed under an optical microscope. Although there were some beads in irregular oval and tailed shapes, the majority of beads had achieved the desirable spherical shapes. The result is in line with Martín et al. [32] who reported the production of uniform round beads can be achieved by having consistency in preparing the same concentration and viscosity of sodium alginate and calcium chloride solutions. Dias et al. [33] also stated that the co-extrusion technique could produce uniform-shaped beads. The shape of beads could greatly affect the viability of probiotics within the wall material. Broken or irregular capsules surface could lead to protrusion of probiotic cells, thus, lowering the survivability of probiotics in the human gut [32].

The relationship of the addition of wall material and coating material on the size and MEE of microencapsulated LGG were investigated and results were shown in Table 3. Calcium alginate beads were larger in terms of beads size when compared with alginate-starch microcapsules and xanthan gumcoated microcapsules. The findings were in contrast with Zanjani et al. [17] who found that the addition of chitosan and PLL coatings to alginate beads had increased the size of beads. Few parameters such as concentration and types and of coatings, drying, and hardening time of beads and the distance between the encapsulator outlet and hardening solution could affect the size of beads [32]. 
http://wjst.wu.ac.th
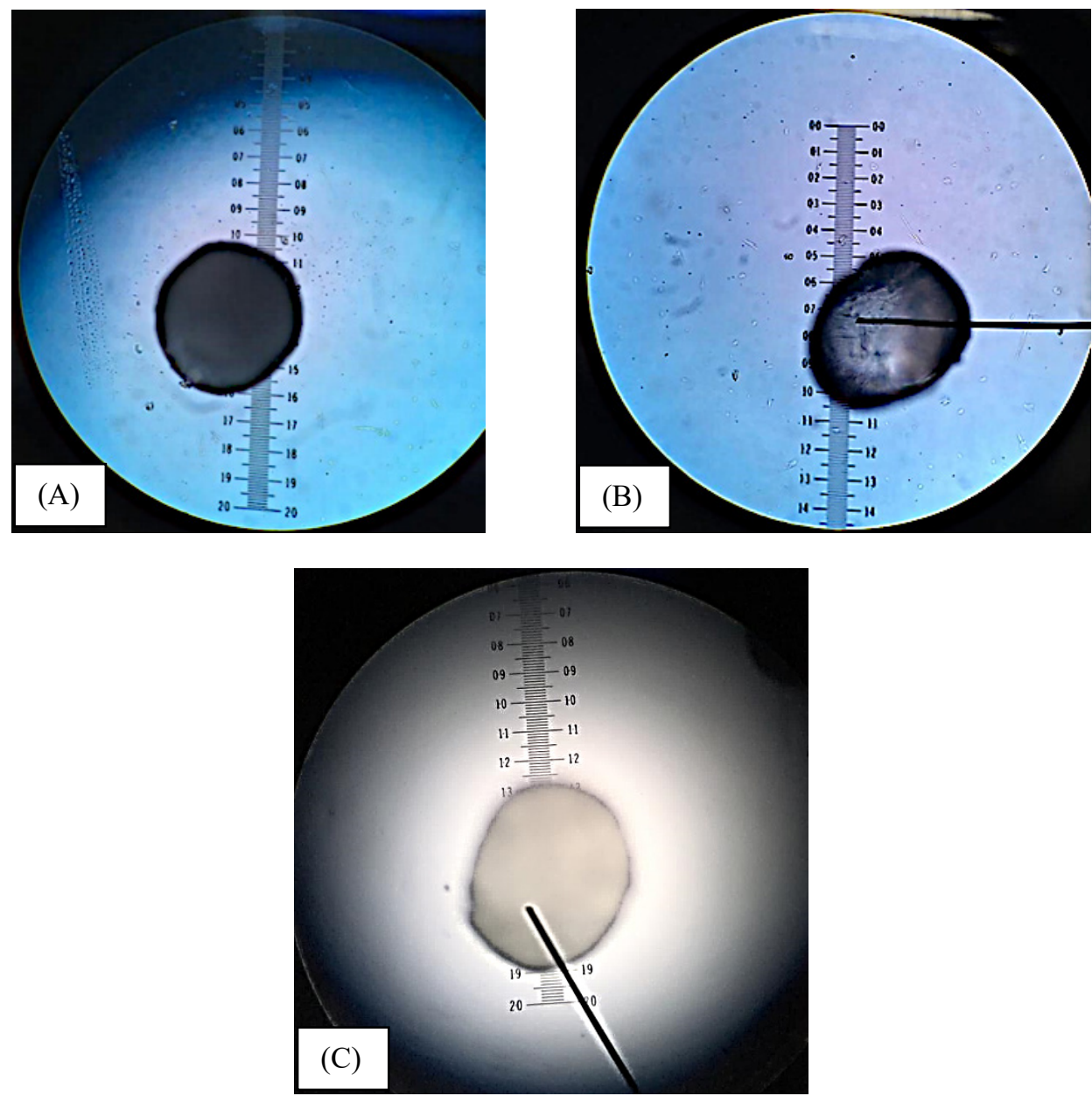

Figure 1 Morphology and size of microcapsules measured with a scale micrometer (A) Calcium-alginate beads, (B) Alginate-starch beads (2\%(w/v) resistant starch), (C) Alginate-starch beads coated with $0.3 \%$ $(\mathrm{w} / \mathrm{v})$ xanthan gum.

On the other hand, the bead size for xanthan gum-coated microcapsules was found to have no significant difference $(p>0.05)$ when compared with alginate-starch microcapsules. This showed that the incorporation of xanthan gum coating did not change the size of beads. The results were in agreement with Fareez et al. [34] who had reported the addition of chitosan coating to alginate-xanthan gum microcapsules did not affect the size of beads. Similarly, Chew et al. [24] and Nualkaekul et al. [19] also reported that probiotic-alginate beads with low coating concentration $(<0.4 \% \mathrm{w} / \mathrm{v})$ were similar in size as compared to uncoated beads. The bead size only increased when a high concentration of coating (1.0 $\% \mathrm{w} / \mathrm{v}$ ) was used in the study [24]. Hence, the possible explanation for the size of coated beads did not vary from the uncoated beads was the concentration of xanthan gum coating used in this study was low.

The size of the beads produced in this study was ranged from 562.67 to $614.33 \mu \mathrm{m}$. The size of beads also has a high impact on the texture of food. Micron bead size can deliver a soft and smooth texture when it is being incorporated into food products when compared with a bead in millimeter sized. However, increment in bead size can improve the protection of probiotics in the gastrointestinal environment. This is due to the formation of cross-linkage between divalent ions and alginate bead core 
http://wjst.wu.ac.th

[36]. Furthermore, bead size below $1,000 \mu \mathrm{m}$ is considered as smooth in terms of texture by consumers upon consumption [37], hence the bead size range in this study is acceptable.

Table 3 Average bead size in diameter and MEE (\%) of LGG.

\begin{tabular}{|c|c|c|c|c|}
\hline Probiotic & Wall material & Coating & $\begin{array}{l}\text { Diameter } \\
(\mu \mathrm{m})\end{array}$ & $\begin{array}{l}\text { Microencapsulation } \\
\text { efficiency (MEE) (\%) }\end{array}$ \\
\hline LGG & Calcium alginate & - & $614.33 \pm 35.35^{b}$ & $25.37 \pm 2.98^{\mathrm{a}}$ \\
\hline LGG & $\begin{array}{l}\text { Calcium alginate- } \\
\text { Resistant starch }\end{array}$ & - & $601.23 \pm 20.12^{\mathrm{a}}$ & $73.65 \pm 2.65^{b}$ \\
\hline LGG & $\begin{array}{l}\text { Calcium alginate- } \\
\text { Resistant starch }\end{array}$ & Xanthan gum & $562.67 \pm 16.02^{\mathrm{a}}$ & $84.67 \pm 2.30^{c}$ \\
\hline
\end{tabular}

In comparison between the MEE for the 3 types of beads, it was shown that the addition of resistant starch and xanthan gum coating had increased the MEE of encapsulated LGG. It was found that calcium alginate beads had the lowest MEE of $25.37 \%$ while xanthan gum-coated beads had the highest MEE of $84.67 \%$. The addition of resistant starch had positive effect in improving the MEE of encapsulated probiotics. This was in agreement with Etchepare et al. [38] who stated the addition of $2 \%$ resistant starch had increased the MEE of bacteria. In addition, xanthan gum-coated beads microcapsules were found to have higher MEE when compared with alginate-starch beads and calcium alginate beads. However, this was in contrast with Chen et al. [21] findings whereby xanthan gum coating reduced the encapsulation efficiency of B. bifidum BB01. This could be due to different encapsulation methods and shell materials used during the microencapsulation process. Nevertheless, the high MEE of LGG microcapsules with resistant starch coated with xanthan gum demonstrated the protective effect of both resistant starch and xanthan gum coating towards the viability of LGG.

\section{Survival of free and microencapsulated Lactobacillus rhamnosus GG under simulated gastrointestinal conditions}

From Figure 2, it was shown that the viable cell counts for all 3 forms of LGG (free cells, encapsulated bacteria with and without xanthan gum coating) decreased when the incubation time increased. During the $2^{\text {nd }}$ hour of incubation, free cells had suffered a sharp decline in viable cell counts when compared with encapsulated bacteria with and without xanthan gum coating. Free cells suffered a $2.41 \log$ reduction while the survival loss for encapsulated bacteria with and without xanthan gum coating was 0.96 and 1.84, respectively. The decreasing trend of free cell, encapsulated bacteria with and without xanthan gum coating were attributed to the acidic environment of gastric juice [28].

After $2 \mathrm{~h}$ of incubation in SGJ, all 3 forms of LGG were transferred to SIJ for further incubation of $4 \mathrm{~h}$. The same trend was observed for the reduction of viable cell counts for all 3 forms of LGG. Free cells had experienced a sharp decline in viable cell counts while encapsulated bacteria with xanthan gum coating had a mild reduction in viable cell counts during the $4^{\text {th }}$ and $6^{\text {th }}$ hours of incubation. Both encapsulated LGG with and without xanthan gum coating had higher viability when compared with free cells. After $4 \mathrm{~h}$ of incubation in SIJ, free cells suffered a $5.47 \mathrm{log}$ reduction while encapsulated bacteria with and without xanthan gum coating had 1.0 and $2.09 \log$ reduction in viability, respectively. 
http://wjst.wu.ac.th

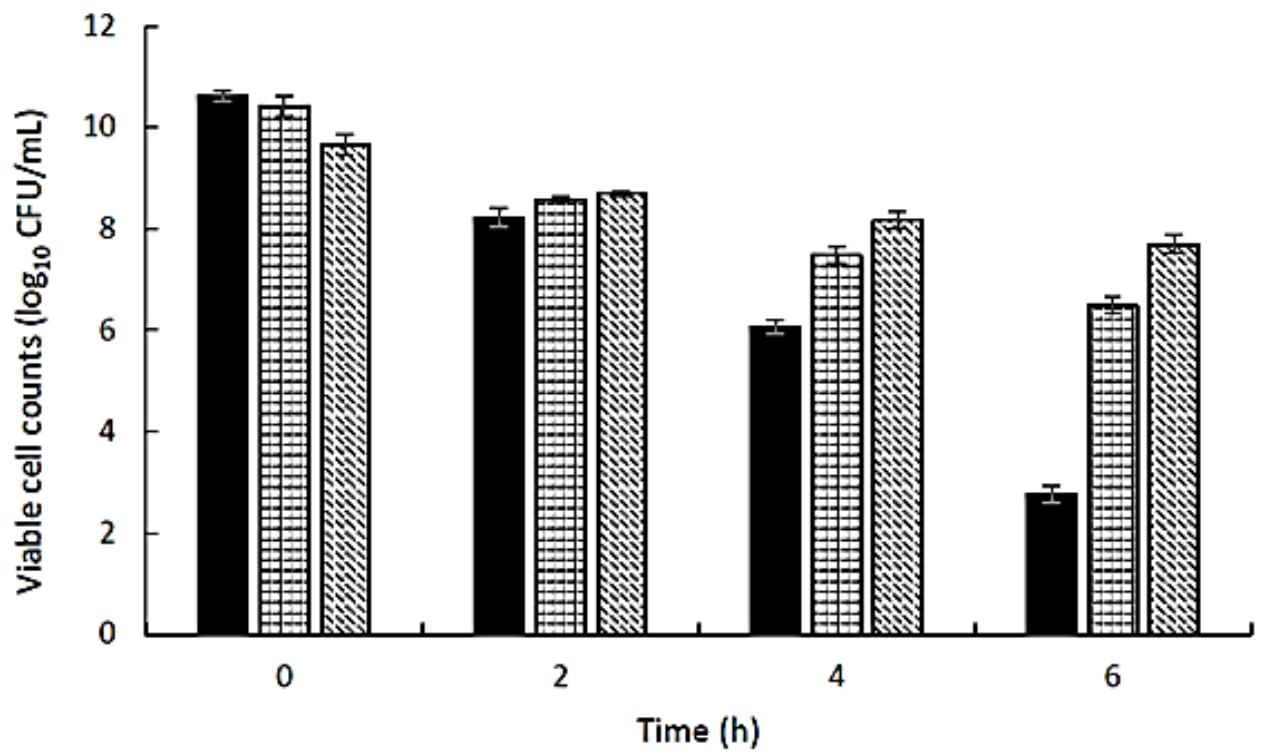

口 Free cell $\boxplus$ Alginate-resistant starch $\mathbf{\Delta A l g i n a t e - r e s i s t a n t ~ s t a r c h - x a n t h a n ~ g u m ~}$

Figure 2 Viable cell counts of free and microencapsulated LGG under sequential digestion. Error bars indicate the standard deviation of triplicate experiments $(n=3)$.

As observed from Figure 2, encapsulated bacteria with and without xanthan gum coating had preserved probiotic viability at the end of sequential digestion. Both formulations had retained the minimum required cell count of $10^{6}-10^{7} \mathrm{CFU} / \mathrm{mL}$. Free cells had experienced a sharp decline in viable cell counts and did not maintain high viability. The viable cell counts of free cells were lower than the recommended amount. It was shown that LGG was sensitive to acidic conditions and bile salt treatment. The findings were in agreement with Karu and Sumeri [39] who reported the viability of LGG had reduced about 4 and $5 \log$ units after incubated in $0.4 \%$ bile.

Better survivability was observed in encapsulated bacteria with and without xanthan gum coating. The results had demonstrated the positive role of resistant starch in protecting probiotics against adverse conditions. The addition of resistant starch into the alginate matrix was able to increase probiotic survivability in a low $\mathrm{pH}$ environment as the starch particles have high binding capacities that curbed the diffusion of calcium ions at the outside of microcapsules [30].

Furthermore, resistant starch will swell when in contact with water and it was used as a filler material for alginate beads. The swollen starch particles helped to increase the firmness and support the microcapsules structure against adverse conditions like freezing temperature and heat treatment [11]. Moreover, resistant starch is a type of prebiotic which has synergistic effect when added to probiotic and it increased the recovery of encapsulated bacteria [40].

Encapsulated bacteria with xanthan gum coating was the most effective in protecting LGG during the simulated gastrointestinal conditions. On the other hand, encapsulated bacteria without xanthan gum coating could offer protection to probiotics but was less effective without coating material. The results were in agreement with Chen et al. [21] who reported the addition of xanthan gum coating to xanthanchitosan microcapsules had improved Bifidobacterium BB01 survivability during simulated gastrointestinal conditions and storage in yogurt.

Xanthan gum was reported to have high stability over a wide range of $\mathrm{pH}$ range and the viscosity of the gum was slightly affected over high food processing temperature such as sterilization [41]. When xanthan gum was in contact with the acidic solution in gastric juice, the electrostatic linkage between the 
http://wjst.wu.ac.th

carboxyl group and amino groups were disappeared, this resulted in the increased osmotic pressure and swelling in xanthan gum molecules. The swollen xanthan gum molecules had allowed the penetration of water molecules in which acted as a buffer to acidic compounds. This had helped the microcapsules to increase resistance against low $\mathrm{pH}$ gastric fluid [22]. Moreover, bile salts had an ion exchange reaction with xanthan gum-coated microcapsules which decreased the network porosity. Lower network porosity had decreased the diffusion of bile salts into the microcapsule, and thus, it reduced the bacterial stress during simulated intestinal digestion [25].

\section{Conclusions}

Microencapsulation of LGG using calcium alginate-resistant starch with xanthan gum coating was conducted using the co-extrusion method. Concentration of $2.0 \%(\mathrm{w} / \mathrm{v})$ resistant starch and $0.3 \%(\mathrm{w} / \mathrm{v})$ xanthan gum were chosen for microencapsulation due to highest MEE. The beads were in the micron range and had a uniform and spherical shape. Encapsulated LGG had higher survivability under simulated gastrointestinal conditions when compared with free cells. The addition of resistant starch had improved the viability of LGG during gastrointestinal digestion while the incorporation of xanthan gum coating had further enhanced the protective effect to probiotics. Both encapsulated LGG with and without xanthan gum coating had achieved the desired amount of viable cell counts of $10^{6} \mathrm{CFU} / \mathrm{g}$.

\section{Acknowledgements}

This work was supported by the UCSI University Pioneer Scientist Incentive Fund under Grant PSIF Proj-In-FAS-055.

\section{References}

[1] W Krasaekoopt and S Watcharapoka. Effect of addition of inulin and galactooligosaccharide on the survival of microencapsulated probiotics in alginate beads coated with chitosan in simulated digestive system, yogurt and fruit juice. LWT - Food Sci. Technol. 2014; 57, 761-6.

[2] MAK Zanjani, BG Tarzi, A Sharifan and N Mohammadi. Microencapsulation of probiotics by calcium alginate-gelatinized starch with chitosan coating and evaluation of survival in simulated human gastro-intestinal condition. Iran. J. Pharm. Res. 2014; 13, 843-52.

[3] SC Siang, LK Wai, NK Lin and PL Phing. Effect of added prebiotic (isomalto-oligosaccharide) and coating of beads on the survival of microencapsulated Lactobacillus rhamnosus GG. Food Sci. Technol. 2019; 39, 601-9.

[4] C Ceapa, M Davids, J Ritari, J Lambert, M Wels, FP Douillard, T Smokvina, WMD Vos, J Knol and M Kleerebezem. The variable regions of Lactobacillus rhamnosus genomes reveal the dynamic evolution of metabolic and host-adaptation repertoires. Genome Biol. Evol. 2016; 8, 1889-905.

[5] Y Zhang, L Zhang, M Du, H Yi, C Guo, Y Tuo, X Han, J Li, L Zhang and L Yang. Antimicrobial activity against Shigella sonnei and probiotic properties of wild lactobacilli from fermented food. Microbiol. Res. 2011; 167, 27-31.

[6] J Flach, MBVD Waal, AFM Kardinaal, J Schloesser, RMAJ Ruijschop and E Claassen. Probiotic research priorities for the healthy adult population: A review on the health benefits of Lactobacillus rhamnosus GG and Bifidobacterium animalis subspecies lactis BB-12. Cogent Food Agric. 2018; 4, 1452839.

[7] WL Yee, CL Yee, NK Lin and PL Phing. Microencapsulation of Lactobacillus acidophilus NCFM incorporated with mannitol and its storage stability in mulberry tea. Ciênc. Agrotec. 2019; 43, e005819.

[8] SH Al-Sheraji, A Ismail, MY Manap, S Mustafa, RM Yusof and FA Hassan. Prebiotics as functional foods: A review. J. Funct. Foods 2013; 5, 1542-53.

[9] R Valcheva and LA Dieleman. Prebiotics: Definition and protective mechanisms. Best Pract. Res. Clin. Gastroenterol. 2016; 30, 27-37.

[10] ML Marco, D Heeney, S Binda, CJ Cifelli, PD Cotter, B Foligné, M Gänzle, R Kort, G Pasin, A 
http://wjst.wu.ac.th

Pihlanto, EJ Smid and R Hutkins. Health benefits of fermented foods: Microbiota and beyond. Curr. Opin. Biotechnol. 2017; 44, 94-102.

[11] MAK Zanjani, MR Ehsani, BG Tarzi and A Sharifan. Promoting probiotics survival by microencapsualtion with hylon starch and genipin cross-linked coatings in simulated gastrointestinal condition and heat treatment. Iran. J. Pharm. Res. 2018; 17, 753-66.

[12] AK Anal and H Singh. Recent advances in microencapsulation of probiotics for industrial applications and targeted delivery. Trends Food Sci. Technol. 2007; 18, 240-51.

[13] LE Shi, ZH Li, DT Li, M Xu, HY Chen, ZL Zhang and ZX Tang. Encapsulation of probiotic Lactobacillus bulgaricus in alginate - milk microspheres and evaluation of the survival in simulated gastrointestinal conditions. J. Food Eng. 2013; 117, 99-104.

[14] SL Ng, KW Lai, KL Nyam and LP Pui. Microencapsulation of Lactobacillus plantarum 299v incorporated with oligofructose in chitosan coated-alginate beads and its storage stability in ambarella juice. Malays. J. Microbiol. 2019; 15, 408-18.

[15] MP Silva, FL Tulini, MM Ribas, M Penning, CS Fávaro-Trindade and D Poncelet. Microcapsules loaded with the probiotic Lactobacillus paracasei BGP-1 produced by co-extrusion technology using alginate/shellac as wall material: Characterization and evaluation of drying processes. Food Res. Int. 2016; 89, 582-90.

[16] PX Chia, LJ Tan, CMY Huang, EWC Chan and SY Wong. Hydrogel beads from sugar cane bagasse and palm kernel cake, and the viability of encapsulated Lactobacillus acidophilus. EPolymers 2015; 15, 411-8.

[17] MAK Zanjani, MR Ehsani, BG Tarzi and A Sharifan. Promoting Lactobacillus casei and Bifidobacterium adolescentis survival by microencapsulation with different starches and chitosan and poly L-lysine coatings in ice cream. J. Food Process. Preserv. 2018; 42, 1-10.

[18] RR Mokarram, SA Mortazavi, MBH Najafi and F Shahidi. The influence of multi stage alginate coating on survivability of potential probiotic bacteria in simulated gastric and intestinal juice. Food Res. Int. 2009; 42, 1040-5.

[19] S Nualkaekul, D Lenton, MT Cook, VV Khutoryanskiy and D Charalampopoulos. Chitosan coated alginate beads for the survival of microencapsulated Lactobacillus plantarum in pomegranate juice. Carbohydr. Polym. 2012; 90, 1281-7.

[20] GB Brinques and MAZ Ayub. Effect of microencapsulation on survival of Lactobacillus plantarum in simulated gastrointestinal conditions, refrigeration, and yogurt. J. Food Eng. 2011; 103, 123-8.

[21] L Chen, T Yang, Y Song, G Shu and H Chen. Effect of xanthan-chitosan-xanthan double layer encapsulation on survival of Bifidobacterium BB01 in simulated gastrointestinal conditions, bile salt solution and yogurt. LWT - Food Sci. Technol. 2017; 81, 274-80.

[22] H Habibi and K Khosravi-Darani. Effective variables on production and structure of xanthan gum and its food applications: A review. Biocatal. Agric. Biotechnol. 2017; 10, 130-40.

[23] WK Ding and NP Shah. Effect of various encapsulating materials on the stability of probiotic bacteria. J. Food Sci. 2009; 74, M100-7.

[24] SC Chew, CP Tan, K Long and KL Nyam. In-vitro evaluation of kenaf seed oil in chitosan coatedhigh methoxyl pectin-alginate microcapsules. Ind. Crops Prod. 2015; 76, 230-6.

[25] G Shu, Y He, L Chen, Y Song, J Cao and H Chen. Effect of xanthan-chitosan microencapsulation on the survival of Lactobacillus acidophilus in simulated gastrointestinal fluid and dairy beverage. Polymers (Basel) 2018; 10, 588.

[26] Annan NT, AD Borza and LT Hansen. Encapsulation in alginate-coated gelatin microspheres improves survival of the probiotic Bifidobacterium adolescentis $15703 \mathrm{~T}$ during exposure to simulated gastro-intestinal conditions. Food Res. Int. 2008; 41, 184-93.

[27] M Chávarri, I Marañón, R Ares, FC Ibáñez, F Marzo and MDC Villarán. Microencapsulation of a probiotic and prebiotic in alginate-chitosan capsules improves survival in simulated gastro-intestinal conditions. Int. J. Food Microbiol. 2010; 142, 185-9.

[28] MDA Etchepare, GC Raddatz, ÉMDM Flores, LQ Zepka, E Jacob-Lopes, JS Barin, CRF Grosso and CRD Menezes. Effect of resistant starch and chitosan on survival of Lactobacillus acidophilus microencapsulated with sodium alginate. LWT - Food Sci. Technol. 2016; 65, 511-7. 
http://wjst.wu.ac.th

[29] LK Sarao and M Arora. Probiotics, prebiotics, and microencapsulation: A review. Crit. Rev. Food Sci. Nutr. 2017; 57, 344-71.

[30] S Mathews. Microencapsulation of probiotics by calcium alginate and gelatin and evaluation of its survival in simulated human gastro-intestinal condition. Int. J. Curr. Microbiol. Appl. Sci. 2017; 6, 2080-7.

[31] S Sethi, Saruchi, BS Kaith, M Kaur, N Sharma and V Kumar. Cross-linked xanthan gum-starch hydrogels as promising materials for controlled drug delivery. Cellulose 2020; 27, 4565-89.

[32] MJ Martín, F Lara-Villoslada, MA Ruiz and ME Morales. Microencapsulation of bacteria: A review of different technologies and their impact on the probiotic effects. Innov. Food Sci. Emerg. Technol. $2015 ; 27,15-25$.

[33] MI Dias, ICFR Ferreira and MF Barreiro. Microencapsulation of bioactives for food applications. Food Funct. 2015; 6, 1035-52.

[34] IM Fareez, SM Lim, RK Mishra and K Ramasamy. Chitosan coated alginate-xanthan gum bead enhanced $\mathrm{pH}$ and thermotolerance of Lactobacillus plantarum LAB12. Int. J. Biol. Macromol. 2015; 72, 1419-28.

[35] T Riaz, MW Iqbal, M Saeed, I Yasmin, HAM Hassanin, S Mahmood and A Rehman. In vitro survival of Bifidobacterium bifidum microencapsulated in zein-coated alginate hydrogel microbeads. J. Microencapsul. 2019; 36, 192-203.

[36] AD Prisco and G Mauriello. Probiotication of foods: A focus on microencapsulation tool. Trends Food Sci. Technol. 2016; 48, 27-39.

[37] A Nag, KS Han and H Singh. Microencapsulation of probiotic bacteria using pH-induced gelation of sodium caseinate and gellan gum. Int. Dairy J. 2011; 21, 247-53.

[38] MDA Etchepare, JS Barin, AJ Cichoski, E Jacob-Lopes, R Wagner, LLM Fries and CRD Menezes. Microencapsulation of probiotics using sodium alginate. Ciênc. Rural 2015; 45, 1319-26.

[39] R Karu and I Sumeri. Survival of Lactobacillus rhamnosus GG during simulated gastrointestinal conditions depending on food matrix. J. Food Res. 2016; 5, 56.

[40] SA Zaman and SR Sarbini. The potential of resistant starch as a prebiotic. Crit. Rev. Biotechnol. 2016; 36, 578-84.

[41] A Kumar, KM Rao and SS Han. Application of xanthan gum as polysaccharide in tissue engineering: A review. Carbohydr. Polym. 2018; 180, 128-44. 